\title{
Zur Theorie der ganzen homogenen Functionen.
}

(Von Herrn Otto Hesse zu Heidelberg.)

\section{L e h r s a t z.}

"W enn die Determinante einer ganzen homogenen Function der $n$ "Variabeln $x_{1}, x_{2}, \ldots x_{n}$ identisch verschwindet, so läfst sich die „Function durch bestimmte lineare Substitutionen von der Form:

$$
x_{\%}=a_{1}^{*} y_{1}+a_{2}^{x} y_{2}+\cdots+a_{n}^{x} y_{n}
$$

„auf eine Function der Variabeln $y_{1}, y_{2}, \ldots y_{n}$ zurückführen, in wel"cher eine dieser Variabeln fehlt".

Diesen in diesem Journal Bd. 42, p. 119 mitgetheilten Lehrsatz strenger zu begründen ist der Zweck dieser Abhandlung.

Es sei $u$ eine beliebige ganze homogene. Function $m^{\text {ter }}$ Ordnung von den $n$ Variabeln $x_{1}, x_{2}, \ldots x_{n} ;$ ferner seien $u_{1}, u_{2}, \ldots u_{n}$ die ersten, $u_{11}, u_{12}, \ldots u_{n n}$ die zweiten partiellen Differentialquotienten dieser Function nach den Variabeln genommen. Die Determinante der letzteren, gebildet aus den Componenten:

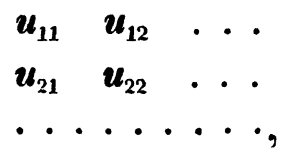

wird die Determinante der Function $u$ genannt, und soll mit dem Zeichen $\Delta$ bezeichnet werden. Obwohl $u_{x \lambda}$ und $u_{\lambda x}$ dem Werthe nach einander gleich sind, so will ich doch bei der partiellen Differentiation von $\Delta$ nach diesen Componenten einen Unterschied machen, um die bekannten Determinantensätze ohne Weiteres in Anwendung bringen zu können.

Dieses vorausgesetzt, hat man bekanntlich

$$
\begin{aligned}
& \Delta=\Delta_{x 1} u_{x 1}+\Delta_{x 2} u_{x 2}+\cdots+\Delta_{x n} u_{x n}, \\
& 0=\Delta_{x 1} u_{\lambda_{1}}+\Delta_{x 1} u_{\lambda 2}+\cdots+\Delta_{x n} u_{\lambda n},
\end{aligned}
$$

wenn man der Kürze wegen setzt $\frac{\partial \Lambda}{\partial u_{x \lambda}}=\Delta_{x \lambda}$. 
Die Determinante $\Delta$ ist in Rücksicht auf die Variabeln von der Ordnung $n(m-2)$, während die partiellen Differentialquotienten $\Delta_{x \lambda}$ derselben sämmtlich von der Ordnung $(n-1)(m-2)$ sind.

Es habe aber die vorhin bezeichnete Function $u$ die Eigenschaft, dafs ihre Determinante $\Delta$ identisch verschwindet, $d$. $h$. für alle Werthe der Variabeln. Diese Annahme wollen wir auch im Folgenden festhalten und untersuchen, welche Folgerungen sich daraus ziehen lassen.

Zunächst bemerke man, dafs die beiden aufgestellten Gleichungen in die eine identische Gleichung übergehen:

$$
\text { (1.) } 0=\Delta_{x 1} u_{\lambda 1}+\Delta_{x 2} u_{\lambda 2}+\cdots+\Delta_{x n} u_{\lambda n} \text {, }
$$

in welcher der Index $\lambda$ alle Zahlen $1,2, \ldots n$ auch $\varkappa$ umfarst. Aus dieser Gleichung entspringt nun ein ganzes System in Rücksicht auf die $n$ partiellen Differentialquotienten $\Delta_{x 1}, \Delta_{x 2}, \ldots \Delta_{x n}$ linearer Gleichungen, wenn man dem Index $\lambda$ die bezeichneten Werthe zuertheilt, welches dazu dienen kann, die Verhältnisse der genannten $n$ partiellen Differentialquotienten zu bestimmen.

Diese $n$ partiellen Differentialquotienten, für welche der Index $*$ unveränderlich aus der Reihe der Zahlen 1, $2, \ldots n$ aber beliebig gewählt ist, können einen allen gemeinsamen Factor $M$ haben von der Ordnung $\mu$ in Rücksicht auf die Variabeln. Haben sie keinen solchen Factor, so ist $M=1$. Wenn nun $\boldsymbol{M}$ der gröfste gemeinsame Factor ist, so können wir setzen :

$$
\text { (2.) } \quad \Delta_{x: 1}=a_{1} M, \quad \Delta_{x 2}=a_{2} M, \ldots \Delta_{x n}=a_{n} M \text {, }
$$

indem wir $a_{1}, a_{2}, \ldots a_{n}$ ganze homogene Functionen bedeuten lassen sämmtlich von der Ordnung $(n-1)(m-2)-\mu$, welche keinen allen gemeinsamen Factor haben. Hiernach werden alle Functionen $a_{1}, a_{2}, \ldots a_{n}$ Constanten, wenn eine derselben eine Constante ist.

Eine Erwähnung verdient der Fall, dafs einer der $n$ Differentialquotienten $\Delta_{x \lambda}$ identisch verschwindet. In diesem Falle verschwinden sämmtliche Differentialquotienten $\Delta_{x \lambda}$, auch die, welche nicht unter den genannten Differentialquotienten enthalten sind, welches sich aus dem Vergleich des Systems Gleichungen (1.) mit dem folgenden:

$$
0=\Delta_{v_{1}} u_{\lambda_{1}}+\Delta_{v_{2}} u_{\lambda_{2}}+\cdots
$$

sogleich ergiebt. Denn da dieses System ebenso zur Werthbestimmung der Verbältnisse von $\Delta_{v_{1}}: \Delta_{v^{2}} \ldots$ dient als jenes zur Werthbestimmung der Verhältnisse von $\Delta_{x 1}: \Delta_{x 2} \ldots$, und beide Systeme abgesehen von der Bezeichnung 
der Unbekannten auf eines hinauskommen, so folgt hieraus:

$$
\Delta_{x 1}: \Delta_{x 2}: \ldots \Delta_{x \mu} \ldots=\Delta_{\nu 1}: \Delta_{\nu 2}: \ldots \Delta_{\nu \mu} \ldots
$$

und wenn $\Delta_{x \mu}$ verschwindet, dafs auch $\Delta_{\nu \mu}=\Delta_{\mu \nu}$ verschwindet. Wir haben also den Satz:

„Wenn die Determinante einer homogenen ganzen Function von $\boldsymbol{n}$ Va„riabeln identisch verschwindet, und wenn aufserdem der partielle Dif„ferentialquotient der Determinante nach einer der Componenten genom„men identisch verșchwindet, so verschwinden auch die übrigen partiellen „Differentialquotienten der Determinante nach den Componenten genommen "identisch."

Es ist dieses der Fall, in welchem die Function $\boldsymbol{u}$ durch lineare Substitutionen neuer Variabeln sich zurückführen läfst auf eine Function, welche zwei Variabeln weniger enthält. Dieser Fall verlangt eine besondere Untersuchung. Wir werden ihn daher hier unberücksichtigt lassen, indem wir festsetzen, dafs weder $M$ noch eine der Gröfsen $a_{1}, a_{2}, \ldots a_{n}$ identisch verschwinde.

Setzt man die Werthe von (2.) in (1.), so erhält man mit Unterdrückung des Factors $\boldsymbol{M}$ folgende identische Gleichung:

$$
0=a_{1} u_{\lambda 1}+a_{2} u_{\lambda 2}+\cdots+a_{n} u_{\lambda_{n}},
$$

welche wieder ein ganzes System identischer Gleichungen repräsentirt, die aus ihr erhalten werden, wenn man dem Index $\lambda$ die Werthe $1,2, \ldots n$ zuertheilt.

Wenn man diese Gleichung mit $x_{\lambda}$ multiplicirt und für $\lambda$ nach einander die eben genannten Werthe setzt, so erhält man mit Berủcksichtigung der identischen Gleichung: $u_{1 \mu} x_{1}+u_{2 \mu} x_{2}+\cdots+u_{n \mu} x_{n}=(m-1) u_{\mu}$ durch Addition folgende ebenfalls identische Gleichung:

$$
\text { (4.) } \quad 0=a_{1} u_{1}+a_{2} u_{2}+\cdots+a_{n} u_{n} \text {. }
$$

Durch Differentiation nach $x_{\lambda}$ erhält man hieraus mit Berücksichtigung der Gleichung (3.):

$$
\text { (5.) } \quad 0=\frac{\partial a_{1}}{\partial x_{2}} u_{1}+\frac{\partial a_{2}}{\partial x_{\lambda}} u_{2}+\cdots+\frac{\partial u_{n}}{\partial x_{2}} u_{n} \text {. }
$$

Diese identische Gleichung repräsentirt ein ganzes System von $\boldsymbol{n}$ in Rücksicht auf $u_{1}, u_{2}, \ldots u_{n}$ linearen Gleichungen, da $\lambda$ alle jene Werthe $1,2, \ldots n$ annehmen kann. Bildet man daher aus den Coefficienten 


$$
\begin{array}{lll}
\frac{\partial a_{1}}{\partial x_{1}} & \frac{\partial a_{2}}{\partial x_{1}} & \ldots \\
\frac{\partial a_{1}}{\partial x_{2}} & \frac{\partial a_{2}}{\partial x_{2}} & \ldots
\end{array}
$$

die Determinante $\boldsymbol{D}$, so hat man die identische Gleichung

$$
\text { (6.) } \boldsymbol{D}=0 \text {, }
$$

woraus wiederum, wenn man setzt: $\frac{\partial \boldsymbol{D}}{\partial\left(\frac{\partial a_{\nu}}{\partial x_{\mu}}\right)}=\boldsymbol{D}_{\nu \mu}$, folgende identische Gleichung hervorgeht:

$$
\boldsymbol{0}=\boldsymbol{D}_{\mu 1} \frac{\partial \boldsymbol{a}_{v}}{\partial x_{1}}+\boldsymbol{D}_{\mu 2} \frac{\partial \boldsymbol{a}_{\nu}}{\partial x_{2}}+\cdots+\boldsymbol{D}_{\mu n} \frac{\partial \boldsymbol{a}_{\nu}}{\partial x_{n}},
$$

in welcher $\mu$ und $\nu$ irgend welche Zahlen $1,2, \ldots n$ bedeuten, selbst gleiche.

Durch Differentiation der Gleichung (3.) nach $x_{v}$ erhält man:

$$
\mathbf{0}=\left(\frac{\partial \boldsymbol{a}_{1}}{\partial x_{\nu}} \boldsymbol{u}_{\lambda 1}+\frac{\partial \boldsymbol{a}_{2}}{\partial x_{\nu}} \boldsymbol{u}_{\lambda 2}+\cdots+\frac{\partial \boldsymbol{a}_{n}}{\partial \boldsymbol{x}_{\nu}} \boldsymbol{u}_{\lambda n}\right)+\left(\boldsymbol{a}_{1} \boldsymbol{u}_{\lambda 1 \nu}+\boldsymbol{a}_{2} \boldsymbol{u}_{\lambda 2 \nu}+\cdots+\boldsymbol{a}_{n} \boldsymbol{u}_{\lambda n v}\right),
$$

wenn man den dritten Differentialquotienten der Function $u$ nach $x_{\lambda}, x_{\mu}, x_{v}$ mit $\boldsymbol{u}_{\lambda \mu \nu}$ bezeichnet. Setzt man der Kürze wegen :

$$
\boldsymbol{a}_{1} \boldsymbol{u}_{\lambda_{1}}+\boldsymbol{a}_{2} \boldsymbol{u}_{\lambda 2 \nu}+\cdots+\boldsymbol{a}_{n} \boldsymbol{u}_{\lambda_{n \nu}}=-\boldsymbol{w}_{\lambda_{\nu}},
$$

wobei zu bemerken, dafs $w_{\lambda \nu}=w_{\nu \lambda}$, so stellt sich die vorhergèende identische Gleichung also dar:

$$
\text { (9.) } \frac{\partial a_{1}}{\partial x_{\nu}} \boldsymbol{u}_{\lambda 1}+\frac{\partial a_{2}}{\partial x_{\nu}} \boldsymbol{u}_{\lambda 2}+\cdots+\frac{\partial a_{n}}{\partial x_{\nu}} \boldsymbol{u}_{\lambda n}=\boldsymbol{w}_{\lambda \nu} \text {. }
$$

Multiplicirt man diese Gleichung mit $\boldsymbol{D}_{\mu \nu}$, setzt für $\nu$ nach einander die Zablen 1, 2, .. n und addirt, so verschwindet nach (7.) der linke Theil der Summe aller Gleichungen, und man erhält:

$$
\text { (10.) } \quad 0=\boldsymbol{D}_{\mu 1} \boldsymbol{w}_{\lambda_{1}}+\boldsymbol{D}_{\mu 2} \boldsymbol{w}_{\lambda 2}+\cdots+\boldsymbol{D}_{\mu n} \boldsymbol{w}_{\lambda n} \text {. }
$$

Die aufgestellten Gleichungen dienen nun dazu nachzuweisen, dafs die Functionen $a_{1}, a_{2}, \ldots a_{n}$ Constanten sind.

Aus jeder von den Gleichungen (7.) und (10.) geht ein ganzes System linearer Gleichungen hervor, wenn man für $\mu$ die Zahlen setzt $1,2, \ldots n$. Das erste System dient zur Werthbestimmung der Verhältnisse $\frac{\partial a_{v}}{\partial x_{1}}: \frac{\partial a_{v}}{\partial x_{2}} \ldots$, das andere zur Werthbestimmung der Verhältnisse $w_{\lambda_{1}}: w_{\lambda_{2}} \ldots$ Da aber die Coefficienten der Unbekannten in diesen beiden Systemen linearer Glei- 
chungen übereinstimmen, so müssen die Differentialquotienten von $a_{v}$ den entsprechenden Gröfsen $u$ proportional sein. Es ist mithin :

$$
\text { (11.) } \quad \varrho \frac{\partial a_{v}}{\partial x_{1}}=w_{\lambda 1}, \quad \varrho \frac{\partial a_{v}}{\partial x_{2}}=w_{\lambda 2}, \ldots \rho \frac{\partial a_{v}}{\partial x_{n}}=w_{\lambda_{n}} \text {. }
$$

Es bedeutet hier $\rho$ einen nicht verschwindenden Factor für den Fall, dafs nicht alle $w$, verschwinden. Wenn es also ein $\lambda$ giebt, für welches nicht alle $w$ verschwinden, und wenn über dieses nicht alle Differentialquotienten von $a_{\nu}$ verschwinden, so bestehen jene Gleichungen (11.). Das Verschwinden sämmtlicher Differentialquotienten von $a_{v}$ werden wir nicht voraussetzen, weil wir es eben beweisen wollen. Das Verschwinden aller $w$ verlangt eine besondere Untersuchung, die wir nachfolgen lassen werden.

Multiplicirt man die Gleichungen (11.) der Reihe nach mit $x_{1}, x_{2}, \ldots x_{n}$ und addirt sämmtliche Gleichungen, so erhält man, da $a_{v}$ von der Ordnung $(n-1)(m-2)-\mu$

$$
\{(n-1)(m-2)-\mu\} \cdot \varrho \cdot a_{v}=w_{\lambda 1} x_{1}+w_{\lambda 2} x_{2}+\cdots+w_{\lambda n} x_{n} .
$$

Der rechte Theil dieser Gleichung verschwindet aber, wenn man für die $w$ die Werthe aus (8.) setzt, wodurch er übergeht in den rechten, mit (m-2) multiplicirten Theil der Gleichung (3.). Es mufs also nothwendiger Weise auch einer der Factoren des linken Theiles verschwinden. Da aber weder $\varrho$ noch $a_{v}$ verschwinden, so wird $\mu$ der Grad von $M$

$$
\mu=(n-1)(m-2)
$$

gleich dem Grade der Functionen $A_{x \lambda}$ in (2.). Mithin werden die Functionen $a_{1}, a_{2}, \ldots a_{n}$ Constanten sein.

Wenn zweitens alle $w$ verschwinden, so geht (9.) über in:

$$
\text { (12.) } 0=\frac{\partial a_{1}}{\partial x_{\nu}} \boldsymbol{u}_{\lambda_{1}}+\frac{\partial a_{2}}{\partial x_{\nu}} \boldsymbol{u}_{\lambda_{2}}+\cdots+\frac{\partial a_{n}}{\partial x_{\nu}} \boldsymbol{u}_{\lambda_{n}} \text {. }
$$

Diese Gleichung, gleich wie die Gleichung (3.), repräsentirt ein ganzes System linearer Gleichungen, da $\lambda$ die Zahlen $1,2, \ldots n$ bedeutet. Aus dem Vergleich der beiden Systeme ergiebt sich nun durch eine schon angewendete Schlufsfolge:

$$
\text { (13.) } \quad p a_{1}=\frac{\partial a_{1}}{\partial x_{v}}, \quad p a_{2}=\frac{\partial a_{2}}{\partial x_{v}}, \quad \ldots \quad p a_{n}=\frac{\partial a_{n}}{\partial x_{v}},
$$

wo $p$ eine noch zu bestimmende Function der Variabeln $x_{1}, \ldots x_{n}$ bedeutet. Durch Integration erhält man:

(14.) $\quad a_{1}=e^{\int p d x_{\nu}} \cdot C_{1}, \quad a_{2}=e^{\int p d x_{\nu}} \cdot C_{2}, \quad \ldots \quad a_{n}=e^{\int p d x_{\nu}} \cdot C_{n}$. 
Die Crastanten der Integration $\boldsymbol{C}_{1}, \boldsymbol{C}_{2}, \ldots \boldsymbol{C}_{n}$ sind im Allgemeinen Functionen, wetche lie eine Variable $x_{v}$ nicht enthalten.

Die Function an $a ., a_{2}, \ldots a_{n}$ haben hiernach den gemeinsamen Factor $e^{j \neq d x_{r}}$. Da sie aber der Annahme nach keinen solchen Factor haben, so n ufs $y=0$ sein. Mithin verschwinden nach (13.) die Differentialquotienten der genannten Functionen nach $x_{\nu}$ genommen, und da $\nu$ eine beliebige von den Zahlen 1, 2, ...n ist, so verschwinden auch die Differentialquotienten der Functionen $a_{1}, a_{2}, \ldots a_{n}$ nach irgend einer der Variabeln genommen. Sie sind mithin Constanten. Mit Rücksicht auf (4.) hat man daher den Lehrsatz:

„Wenn die Determinante einer homogenen ganzen Function von $\boldsymbol{n}$ Va„riabeln identisch verschwindet, so giebt es immer $n$ Constanten, mit „welchen die ersten partiellen Differentialquotienten der Function zu mul"tipliciren sind, damit die Summe dieser Producte identisch verschwinde". Wie die $n$ Constanten bestimmt werden können, lehren die Gleichungen (2.).

Ich behaupte nun, dafs durch die Substitutionen:

$$
\begin{gathered}
x_{1}=z_{1}+\lambda a_{1}, \\
x_{2}=z_{2}+\lambda a_{2}, \\
\cdots \cdots \\
x_{n}=z_{n}+\lambda a_{n},
\end{gathered}
$$

wo $z_{1}, z_{2}, \ldots z_{n}$ beliebige lineare Functionen $\operatorname{der}(n-1)$ neuen Variabeln $y_{1}, y_{2}, \ldots y_{n-1}$ von der Form $z_{x}=a_{1}^{x} y_{1}+a_{2}^{x} y_{2}+\cdots+a_{n-1}^{x} y_{n-1}$ sind, und $\lambda$ die $n^{\text {te }}$ neue Variable, aus der Function $u$ die letzte Variable ganz verschwindet. In der That: macht man in der Function $u$ die angegebenen Substitutionen, so erhält man durch Entwicklung nach Potenzen von $\lambda$ :

$$
\boldsymbol{u}=\boldsymbol{w}+\lambda \boldsymbol{w}^{\prime}+\frac{\lambda^{2}}{1.2} \boldsymbol{w}^{\prime \prime}+\cdots+\frac{\lambda^{m}}{1.2 \ldots m} \boldsymbol{w}^{(m)} \text {. }
$$

In dieser Entwicklung bedeuten $w$ und $\boldsymbol{w}^{\prime}$ die Ausdrücke, in welche $u$ und $a_{1} u_{1}+a_{2} u_{2}+\cdots+a_{n} u_{n}{ }^{\prime}$ übergehen, wenn man in denselben $z_{1}, z_{2}, \ldots z_{n}$ statt $x_{1}, x_{2}, \ldots x_{n}$ setzt. Ferner ist:

$$
\begin{aligned}
& \boldsymbol{w}^{\prime \prime}=\frac{\partial w^{\prime}}{\partial z_{1}} a_{1}+\frac{\partial w^{\prime}}{\partial z_{z}} a_{2}+\cdots+\frac{\partial w^{\prime}}{\partial z_{n}} a_{n}, \\
& w^{(m)}=\frac{\partial w^{(m-1)}}{\partial z_{1}} a_{1}+\frac{\partial w^{(m-1)}}{\partial z_{2}} a_{2}+\cdots+\frac{\partial w^{(m-1)}}{\partial z_{n}} a_{n} .
\end{aligned}
$$


Da aber der Ausdruck $a_{1} u_{1}+a_{2} u_{2}+\cdots+a_{n} u_{n}$ identisch verschwindet, so verschwindet auch $w^{\prime}$ identisch für alle Werthe von $z_{1}, z_{2}, \ldots z_{n^{*}}$ Verschwindet aber $w^{\prime}$ identisch, so verschwinden auch die partiellen Differentialquotienten dieser Function nach $z_{1}, z_{2}, \ldots z_{n}$ genommen identisch; mithin auch $w^{\prime \prime}$ etc. Es verschwinden alle Functionen $w^{\prime}, w^{\prime \prime}, \ldots w^{(m)}$, und die obige Entwicklung giebt :

$$
\boldsymbol{u}=\boldsymbol{w}
$$

einen Ausdruck für $u$, welcher die $n^{\text {te }}$ neue Variabele $\lambda$ nicht mehr enthält. Heidelberg im December 1858. 